\title{
稠合四环噻嗪烷-4-酮衍生物的合成及其抗肿瘤活性
}

\author{
牛丽萍邢顺凯李小六* 陈 华*
}

(河北大学化学与环境科学学院 河北省化学生物学重点实验室 保定 071002)

\begin{abstract}
摘要 以叔丁氧羰基 $(\mathrm{Boc})$ 保护的脯氨醛 1 、氨基酸酯盐酸盐 $\mathbf{2 a} \sim 2 \mathrm{~d}$ 和巯基水杨酸 $\mathbf{3 a} \sim \mathbf{3 b}$ 为原料，三组分一锅法得到 苯并噻嗪烷-4-酮中间体. 酸性条件下脱除 Boc, 分子内酰胺缩合制备稠合四环噻嗪烷-4-酮衍生物 6 11. 新生成手性碳 (1-C)的构型通过 H-1 和 H-2 的偶合常数及 X-ray 单晶衍射确定. 测试了化合物抗 Hela 和 A549 的肿瘤细胞增殖活性. 结 果表明, 部分化合物具有中等的抗 Hela 细胞活性, 其中 $(13 \mathrm{a} R, 13 \mathrm{~b} R)-1,2,3,13 \mathrm{~b}$-四氢苯并 $[e]$ 吡咯并 $\left[2^{\prime}, 1^{\prime}: 3,4\right]$ 吡嗪并 $[2,1-b][1,3]$ 噻嗪-5,8(6H,13aH)-二酮 $(6 \mathbf{b})$ 的 $\mathrm{IC}_{50}$ 值为 $9.50 \mu \mathrm{mol} / \mathrm{L}$. 所有化合物对 $\mathrm{A} 549$ 的细胞没有抑制活性.
\end{abstract}

关键词 稠合衍生物; 噻嗪烷-4-酮; 三组分缩合; 酰胺环缩合; 抗肿瘤活性

\section{Synthesis of the Fused Tetracyclic Thiazinan-4-one Derivatives and Their Anti-tumor Activitiy}

\author{
Niu, Liping Xing, Shunkai Li, Xiaoliu* Chen, Hua* \\ (Key Laboratory of Chemical Biology of Hebei Province, College of Chemistry and Environmental Science, Hebei University, \\ Baoding 071002)
}

\begin{abstract}
The benzothiazin-4-one intermediates were prepared by the one-pot three-components condensation from the $N$-Boc- $L$-prolinal 1, amino acid ethyl/methyl ester hydrochlorides $\mathbf{2 a} \sim \mathbf{2 d}$, and mercaptobenzoic acids $\mathbf{3 a} \sim \mathbf{3 b}$. After removal of Boc, the target novel fused tetracyclic thiazinan-4-one derivatives $\mathbf{6} \sim \mathbf{1 1}$ were afforded by the intramolecular cyclo-amidation reaction. The absolute configurations of the newly generated chiral carbon (1-C) were determined by the coupling constants of H-1 and H-2 and the X-ray crystallographic structures. The tetracyclic alkaloids were examined for their anti-proliferative activity against Hela and A549 tumor cells. The results showed that some compounds could moderately inhibit the growth of Hela cells, and among them, $(13 \mathrm{a} R, 13 \mathrm{~b} R)-1,2,3,13 \mathrm{~b}$-tetrahydrobenzo[ $e]$ pyrrolo[ $\left[2 ', 1^{\prime}: 3,4\right]$ pyrazino[2,1$b][1,3]$ thiazine-5,8$(6 H, 13 \mathrm{a} H)$-dione $(6 \mathbf{b})$ was the best one with the $\mathrm{IC}_{50}$ value of $9.50 \mu \mathrm{mol} / \mathrm{L}$. However, all the compounds showed no anti-tumor activity against A549.
\end{abstract}

Keywords fused heterocyclic derivatives; thiazinan-4-one; three-components condensation; cyclo-amidation; anti-tumor activity

生物碱(alkaloids)是指存在于生物体内的含氮化合 物，因其种类繁多、结构迥异，并且具有各种各样的生 物活性而备受关注 ${ }^{[1,2]}$. 其中, 具有稠合多环结构的生物 碱和含氮衍生物, 因其独特的结构骨架以及显著的药理 活性, 一直是有机化学(全合成)及药物化学领域的热点 与重点 ${ }^{[3,4]}$.

噻嗪烷-4-酮(图 1)是一类含 $\mathrm{N}$ 和 $\mathrm{S}$ 杂原子的六元环 内酰胺药效团 ${ }^{[5]}$, 其衍生物多具有消炎 ${ }^{[6]}$ 、抗菌 ${ }^{[7]}$ 、抗结

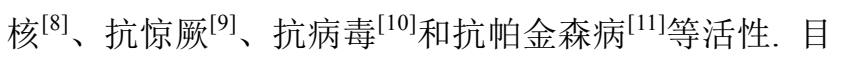

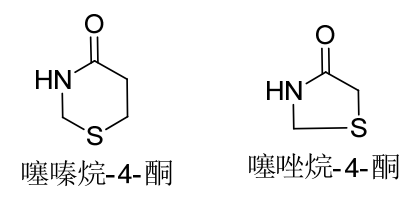

图 1 噻嗪烷-4-酮和噻唑烷-4-酮

Figure 1 Thiazinan-4-one and thiazolidin-4-one

前对噻嗪烷-4-酮的研究并不多, 而含有噻嗪烷-4-酮结 构的稠合多环衍生物更是鲜有报道. 合成结构新颖的含 氮稠合多环衍生物(生物碱), 研究其生物(药理)活性,

\footnotetext{
* Corresponding authors. E-mail: lixl@hbu.cn; hua-todd@163.com

Received July 24, 2018; revised August 23, 2018; published online October 26, 2018.

Project supported by the National Natural Science Foundation of China (No. 21372060) and the Natural Science Fundation of Hebei Province (No. B2016201031).

国家自然科学基金(No. 21372060)和河北省自然科学基金重点(No. B2016201031)资助项目.
} 
对于以结构多样性为导向的新药研发, 具有重要的意 $义^{[12,13]}$.

在此, 作为本实验室长期从事噻唑烷-4-酮 ${ }^{[14]}$ (图 1, 含 $\mathrm{N}$ 和 $\mathrm{S}$ 杂原子的五元环内酰胺)衍生物的合成及其生 物活性研究工作的延续 ${ }^{[9,15 ~ 17]}$, 本文以叔丁氧羰基(Boc) 保护的脯氨醛、氨基酸酯盐酸盐和放基水杨酸为起始原 料, 经三组分一锅法、脱 Boc 保护基和分子内酰胺缩合 三步反应，合成了系列新型的稠合四环噻嗪烷-4-酮衍 生物(Scheme 1), 并测试了所合成化合物的抗肿瘤细胞 增殖活性.
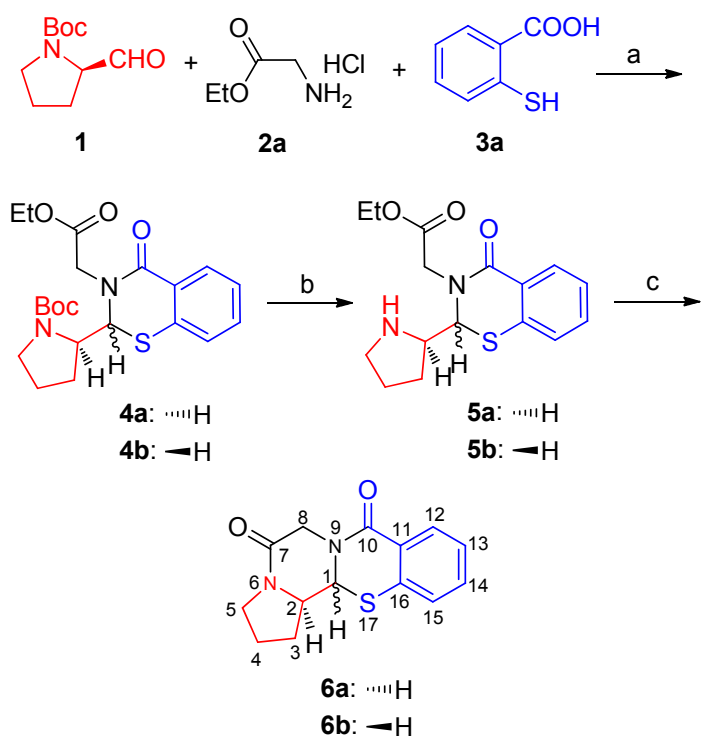

图式 1 稠合四环噻嗪烷-4-酮衍生物的合成

Scheme 1 Synthesis of the fused tetracyclic thiazinan-4-one derivatives

\section{1 结果与讨论}

\section{1 稠合四环噻嗪烷-4-酮衍生物的合成}

因与噻唑烷-4-酮结构相似，噻嗪烷-4-酮骨架的构 建常通过醛、胺、颈基丙酸三组分一锅法反应合成 ${ }^{[18]}$. 参照实验室已有的合成方法 ${ }^{[19,20]}$, 以化合物 $\mathbf{6 a}$ 和 $\mathbf{6 b}$ 的 合成为例, 反应首先是以 $N$-Boc- $L-$ 脯氨醛 $(1)$ 、甘氨酸甲 酯盐酸盐 (2a) 和颈基水杨酸 (3a) 为原料, 并在缩合剂 $N, N^{\prime}$-二环己基碳酰二亚胺(DCC)和催化剂 4-二甲氨基 吡啶(DMAP)的作用下，微波辐射合成了含噻嗪烷-4-酮 结构的中间体 $(\mathbf{4 a}$ 和 $\mathbf{4 b}$, 一对非对映异构体, 比例 1 : 1). 反应的历程是醛和氨基酸形成亚胺，继而颈基水杨 酸对亚胺亲核加成并关环(Scheme 2). 经条件优化, 确 定以甲苯(toluene)为溶剂, 碳酸钾 $\left(\mathrm{K}_{2} \mathrm{CO}_{3}\right)$ 为缚酸剂(中 和氨基酸酯的盐酸, 以使氨基裸露, 利于反应), 温度为 $50{ }^{\circ} \mathrm{C}$ ，微波辐射反应，总收率为 $33 \%$. 反应收率不高， 主要是因为醛和颈基水杨酸直接缩合, 生成了相应的内 酯副产物(Scheme 2) ${ }^{[16]}$, 造成醛原料的消耗.
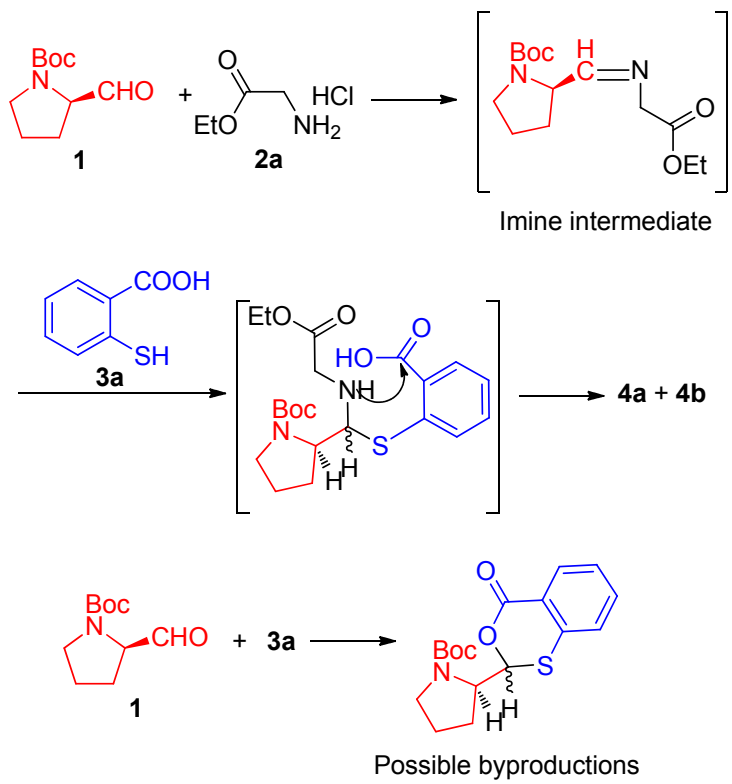

图式 2 中间体 $\mathbf{4 a}$ 和 $\mathbf{4 b}$ 的合成反应机理

Scheme 2 Mechanism of the synthesis of intermediates $4 \mathbf{a}$ and 4b

继而, 以分离到的纯净化合物 $\mathbf{4 a}$ 为原料, 在酸性条 件下，脱除 Boc 保护基得到化合物 5a，进一步在碱性条 件下，经分子内酰胺关环合成目标的稠合四环噻嗪烷4-酮衍生物 6a (Scheme 1). 我们重点探讨了反应溶剂、 碱以及反应温度等条件对酰胺关环反应的影响(表 1). 如表 1 所示, 不同溶剂对反应影响较大(Entries 1 3), 二氯甲烷 $(\mathrm{DCM})$ 为溶剂时, 化合物 $\mathbf{5 a}$ 的溶解及反应效 果，均优于甲醇和乙腈为溶剂，表明非质子小极性溶剂 有利于反应的进行. 因催化剂量的碱可有效实现酰胺关 环, 以 DCM 为溶剂, 进一步探讨了不同的有机碱催化 剂对酰胺关环反应的影响(Entries 4 7). 结果表明, 在 加入有机碱后，反应收率提高较大，其中三乙烯二胺 (DABCO)为催化剂时，效果最好. 最后，探讨了不同温 度对反应产率的影响(Entries $8 \sim 10$ ), 发现反应温度为

表 1 分子内酰胺关环反应合成 $6 a$

Table 1 Synthesis of $\mathbf{6 a}$ by intramolecular amidation

\begin{tabular}{ccccc}
\hline Entry & 溶剂 & 碱 & 温度 $/{ }^{\circ} \mathrm{C}$ & 产率 ${ }^{a} / \%$ \\
\hline 1 & $\mathrm{CH}_{3} \mathrm{OH}$ & $-{ }^{b}$ & 40 & 9 \\
2 & $\mathrm{CH}_{2} \mathrm{Cl}_{2}$ & - & 40 & 13 \\
3 & $\mathrm{CH}_{3} \mathrm{CN}$ & - & 40 & 6 \\
4 & $\mathrm{CH}_{2} \mathrm{Cl}_{2}$ & DBN $^{c}$ & 40 & 51 \\
5 & $\mathrm{CH}_{2} \mathrm{Cl}_{2}$ & DIPEA $^{d}$ & 40 & 40 \\
6 & $\mathrm{CH}_{2} \mathrm{Cl}_{2}$ & DMAP & 40 & 53 \\
7 & $\mathrm{CH}_{2} \mathrm{Cl}_{2}$ & DABCO & 40 & 67 \\
8 & $\mathrm{CH}_{2} \mathrm{Cl}_{2}$ & DABCO & 30 & 35 \\
9 & $\mathrm{CH}_{2} \mathrm{Cl}_{2}$ & DABCO & $\mathbf{5 0}$ & $\mathbf{7 5}$ \\
10 & $\mathrm{CH}_{2} \mathrm{Cl}_{2}$ & DABCO & 60 & 55 \\
\hline
\end{tabular}

${ }^{a}$ Isolated yields, ${ }^{b}$ no catalyst, ${ }^{c} \mathrm{DBN}$ : 1,5-diazabicyclo[4.3.0]-5-nonene, ${ }^{d}$ DIPEA: $N, N$-diisopropylethylamine. 
$50{ }^{\circ} \mathrm{C}$ 时，反应收率最高可达 $75 \%$.

在上述条件下，分别以 3-氨基丙酸甲酯盐酸盐 (2b)、 $D / L-2$-甲基甘氨酸甲酯盐酸盐( 2 c 和 $2 d$ )和 5-氟颈 基水杨酸 $(3 \mathbf{b})$ 等为起始原料, 经三步反应, 合成了系列 新型稠合四环噻嗪烷-4-酮衍生物 7 11 (图 2). 在后续的 合成中, 中间体 4 (非对映异构体)不再分离, 均使用其混 合物用于合成. 待得到四环化合物后, 经硅胶柱层析分 离得到最终的纯净化合物, 简化实验操作.<smiles></smiles>

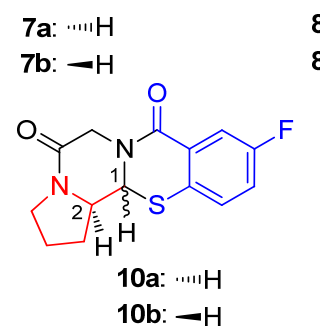<smiles>[CH][C@@]12Sc3ccccc3C(=O)N1[C@@H](C)C(=O)N1CCC[C@H]12</smiles>

8a: $\cdots \cdots \mathrm{H}$

8b: $-\mathrm{H}$

11a: $\cdots \cdot+\mathrm{H}$

11b: $-\mathrm{H}$

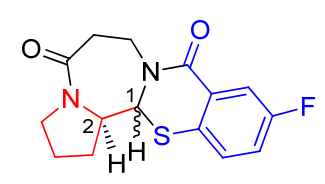

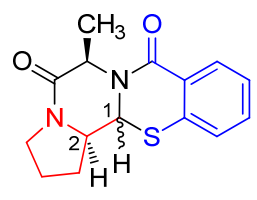

9a: $\cdots+\mathrm{H}$
图 2 稠合四环噻嗪烷-4-酮衍生物

Figure 2 Fused tetracyclic thiazinan-4-one derivatives

\section{2 结构确证}

化合物 6 11 的结构通过 NMR、HR(ESI)MS 等方 法确认. 化合物新生成的手性碳(1-C)的立体构型, 可通 过 H-1 和 H-2 的偶合常数大小确定(表 2). 依据 Karplus 方程, H-1 和 H-2 处于顺式时, 其偶合常数较小; 反式时, 偶合常数较大. 由表 2 可知, $7 \mathbf{a} \sim 11 \mathbf{a}$ 内 $\mathrm{H}-1$ 和 H-2 的偶 合常数较小, 判断其 1-C 的构型为 $S$ 构型, 而具有较大 偶合常数的 $7 \mathrm{~b} \sim 11 \mathrm{~b}$ 为 $R$ 构型. 化合物 $10 \mathrm{a}$ 和 $11 \mathrm{~b}$ 的 X-ray 单晶衍射结构 ${ }^{[21}$ 进一步确证了上述立体构型的判 断(图 3).

表 2 化合物 6 $~ 11$ 中 H-1 和 H-2 的偶合常数

Table 2 Coupling constants of H-1 and H-2 in compounds 6 $\mathbf{1 1}(\mathbf{a} \sim \mathbf{b})$

\begin{tabular}{cc||cc}
\hline Compd. & $J / \mathrm{Hz}$ & Compd. & $J / \mathrm{Hz}$ \\
\hline cis-6a $(S)$ & 4.2 & trans-6b $(R)$ & 10.2 \\
cis-7a $(S)$ & 1.8 & trans-7b $(R)$ & 8.4 \\
cis-8a $(S)$ & 4.2 & trans-8b $(R)$ & 10.2 \\
cis-9a $(S)$ & 4.2 & trans-9b $(R)$ & 10.2 \\
cis-10a $(S)$ & 4.2 & trans-10b $(R)$ & 10.2 \\
cis-11a $(S)$ & 1.8 & trans-11b $(R)$ & 8.4 \\
\hline
\end{tabular}

\section{3 化合物抗肿瘤活性评价}

对所合成化合物 6 11 分别进行了体外抑制人宫颈 癌(Hela)和肺癌(A549)细胞活性测试, 顺铂为阳性对照, 结果见表 3. 总体而言, 所测试化合物对 Hela 细胞增殖

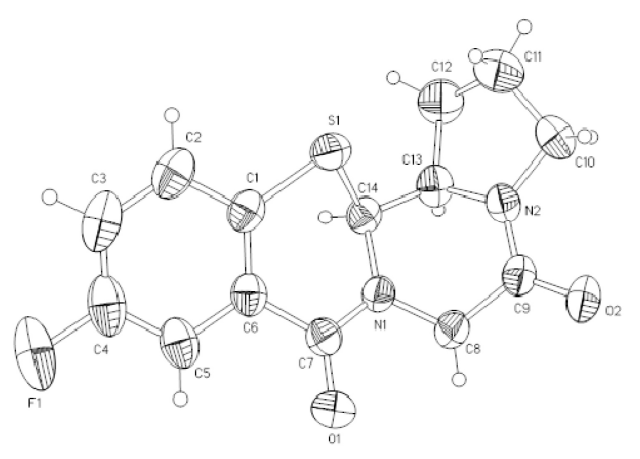

$10 \mathrm{a}$

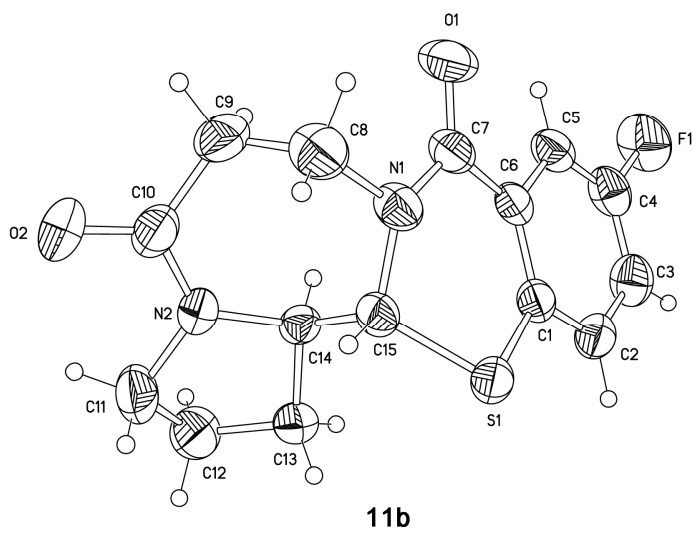

图 3 化合物 $10 \mathrm{a}$ 和 $11 \mathrm{~b}$ 的单晶衍射结构

Figure 3 X-ray crystallographic structures of 10a and 11b

表 3 化合物 6 11 的抗肿瘤活性

Table 3 Antitumor activities of compounds $\mathbf{6} \sim \mathbf{1 1}$

\begin{tabular}{ccc}
\hline \multirow{2}{*}{ Compd. } & \multicolumn{2}{c}{$\mathrm{IC}_{50} /\left(\mu \mathrm{mol} \cdot \mathrm{L}^{-1}\right)$} \\
\cline { 2 - 3 } & Hela & $\mathrm{A} 549$ \\
\hline $\mathbf{6 a}$ & $34.16 \pm 0.05$ & $>50$ \\
$\mathbf{6 b}$ & $\mathbf{9 . 5 0} \pm \mathbf{0 . 1 2}$ & $>50$ \\
$\mathbf{7 a}$ & $20.27 \pm 0.12$ & $>50$ \\
$\mathbf{7 b}$ & $>50$ & $>50$ \\
$\mathbf{8 a}$ & $49.37 \pm 0.21$ & $>50$ \\
$\mathbf{8 b}$ & $>50$ & $>50$ \\
$\mathbf{9 a}$ & $26.41 \pm 0.23$ & $>50$ \\
$\mathbf{9 b}$ & $46.24 \pm 0.21$ & $>50$ \\
$\mathbf{1 0 a}$ & $37.23 \pm 0.15$ & $28.31 \pm 0.04$ \\
$\mathbf{1 0 b}$ & $17.73 \pm 0.04$ & $>50$ \\
$\mathbf{1 1 a}$ & $28.87 \pm 0.10$ & $>50$ \\
$\mathbf{1 1 b}$ & $29.75 \pm 0.08$ & $>50$ \\
顺铂 & $5.87 \pm 0.23$ & $8.20 \pm 0.25$ \\
\hline
\end{tabular}

具有中等偏弱的抑制活性，个别化合物 $6 \mathbf{b}$ 半抑制浓度 $\left(\mathrm{IC}_{50}\right)$ 为 $9.50 \mu \mathrm{mol} / \mathrm{L}$; 而化合物对 $\mathrm{A} 549$ 细胞增殖没有抑 制作用，表现出一定的细胞选择性. 以上活性数据均小 于阳性对照顺铂相应的活性. 此外, 新生成手性碳的立 体构型对化合物抗肿瘤细胞增殖活性影响不大. 


\section{2 结论}

以 Boc 保护的脯氨醛、氨基酸酯盐酸盐和颈基水杨 酸为起始原料, 经三步反应合成了新型稠合四环噻嗪 烷-4-酮衍生物 6 11, 三步总收率为 9\% 24\%. 化合物 的结构通过 NMR 和 HR(ESI)MS 确定. 新生成手性碳 (1-C) 的构型通过化合物中 $\mathrm{H}-1$ 和 H-2 的偶合常数大小以 及 $10 \mathrm{a}$ 和 $11 \mathrm{~b}$ 的 X-ray 单晶衍射确定. 测试了化合物抗 Hela 和 A549 的肿瘤细胞增殖活性. 结果表明, 部分化 合物具有中等的抗 Hela 细胞活性, 其中 $\mathbf{6 b}$ 的 $\mathrm{IC}_{50}$ 值为 $9.50 \mu \mathrm{mol} / \mathrm{L}$, 但所有化合物对 A549 细胞没有抑制活性.

\section{3 实验部分}

\section{1 仪器与试剂}

熔点由 $\mathrm{SGW}^{\circledR} \mathrm{X}-4$ 显微熔点仪(温度计未校正) 测定; 微波反应釜为美国 CEM 公司产品; 核磁共振谱用 BRUKER AC-P600 (600 MHz)型核磁共振仪测定, TMS 为内标; 质谱(ESI)用 Agilent G6300 离子阱液相质谱联 用仪; 高分辨质谱(ESI)用 FTICR-MS (Ionspec 7.0T) 型 质谱仪测定; 旋光仪为 SGW-1 型自动旋光仪, 上海精密 科学仪器有限公司; 单晶衍射仪为 BRUKER Axssmart apexII Mo 靶单晶衍射仪; 层析用硅胶(200 300 目)为 青岛海洋化工厂产品. 本文所用其他试剂均为分析纯, 无水试剂均按常规方法处理, 水为二次蒸馏水.

\section{2 实验方法}

\subsection{1 中间体 $4 \mathrm{a}$ 和 $4 \mathrm{~b}$ 的合成}

于 $25 \mathrm{~mL}$ 茄形烧瓶中加入 $N$-Boc- $L$-脯氨醛 $(94 \mathrm{mg}$, $0.47 \mathrm{mmol}, 1.2$ equiv.)和甘氨酸乙酯盐酸盐 $(54 \mathrm{mg}, 1.0$ equiv.)和 $\mathrm{K}_{2} \mathrm{CO}_{3}(1.0$ equiv.)粉末, 用 $10 \mathrm{~mL}$ 新蒸甲苯溶 解, 微波辐射, $50{ }^{\circ} \mathrm{C}$ 封管反应 $15 \mathrm{~min}$. 薄层色谱(TLC) 监测, 原料脯氨醛反应完全, 加入統基水杨酸 $(120 \mathrm{mg}$, 2.0 equiv.)、 $N, N^{\prime}$-二环已基碳酰二亚胺(DCC, $96.5 \mathrm{mg}, 1.2$ equiv.)和催化剂 4-二甲氨基吡啶(DMAP, $9.45 \mathrm{mg}, 0.2$ equiv.), 继续微波辐射, $50{ }^{\circ} \mathrm{C}$ 反应 $20 \mathrm{~min}$. 反应结束后, 加 $\mathrm{Na}_{2} \mathrm{CO}_{3}$ 粉末中和反应液至中性, 过滤掉固体物质并 用少量乙醚洗涤, 滤液减压浓缩, 残留物经硅胶 $(300 \sim$ 400 目)柱色谱分离 [淋洗剂为 $V$ (石油醚) : $V$ (乙酸乙 酯 $)=6: 1$ ], 得到一对非对映异构体化合物 $4 \mathbf{a}$ 和 $4 \mathbf{b}$ $(1: 1)$, 收率 $33 \%$.

(2S)-2-[(R)-叔丁氧羰基吡咯烷-2-基]-3-(2-乙氧基-2氧亚基乙基)- $2 H$-苯并 $[e][1,3]$ 噻嗪-4-(3H)-酮(4a): 黄色 油状物. ${ }^{1} \mathrm{H}$ NMR $\left(600 \mathrm{MHz}, \mathrm{CD}_{3} \mathrm{OD}\right) \delta: 7.89$ (d, $J=7.8$ $\mathrm{Hz}, 1 \mathrm{H}), 7.42(\mathrm{~d}, J=6.6 \mathrm{~Hz}, 1 \mathrm{H}), 7.30(\mathrm{~d}, J=7.8 \mathrm{~Hz}, 1 \mathrm{H})$, 7.25 (d, $J=7.2 \mathrm{~Hz}, 1 \mathrm{H}), 5.12 \sim 4.92(\mathrm{~m}, 1 \mathrm{H}), 4.80(\mathrm{~d}, J=$ $17.4 \mathrm{~Hz}, 1 \mathrm{H}), 4.46 \sim 4.29(\mathrm{~m}, 1 \mathrm{H}), 4.22(\mathrm{~d}, J=6.6 \mathrm{~Hz}$,
2H), $3.98(\mathrm{~d}, J=17.4 \mathrm{~Hz}, 1 \mathrm{H}), 3.36 \sim 3.26(\mathrm{~m}, 1 \mathrm{H}), 2.77$ $(\mathrm{td}, J=10.5,5.5 \mathrm{~Hz}, 1 \mathrm{H}), 2.19 \sim 1.85(\mathrm{~m}, 2 \mathrm{H}), 1.73(\mathrm{~s}$, 2H), 1.42 (s, 5H), 1.37 (s, 4H), 1.29 (t, $J=7.2 \mathrm{~Hz}, 3 \mathrm{H})$; MS (ESI) $m / z$ : $444.1\left([\mathrm{M}+\mathrm{Na}]^{+}\right)$; HR-ESI-MS calcd for $\mathrm{C}_{21} \mathrm{H}_{28} \mathrm{~N}_{2} \mathrm{O}_{5} \mathrm{SNa}[\mathrm{M}+\mathrm{Na}]^{+}$443.1616, found 443.1613.

$(2 R)$-2-[(R)-叔丁氧羰基吡咯烷-2-基]-3-(2-乙氧基2-氧亚基乙基)- $2 H$-苯并 $[e][1,3]$ 噻嗪-4-( $3 \mathrm{H}$ )-酮 $(4 \mathbf{b})$ : 黄 色油状物, ${ }^{1} \mathrm{H}$ NMR (600 MHz, $\left.\mathrm{CD}_{3} \mathrm{OD}\right) \delta: 8.00$ (d, $J=7.8$ $\mathrm{Hz}, 1 \mathrm{H}), 7.46$ (brs, 1H), 7.34 7.30 (m, 2H), 4.66 4.60 (m, 2H), $4.23 \sim 4.19$ (m, 3H), 3.89 (brs, 1H), 3.52 3.34 $(\mathrm{m}, 2 \mathrm{H}), 2.08 \sim 1.94(\mathrm{~m}, 2 \mathrm{H}), 1.87 \sim 1.84(\mathrm{~m}, 2 \mathrm{H}), 1.42(\mathrm{~d}$, $J=10.8 \mathrm{~Hz}, 9 \mathrm{H}), 1.28$ (t, $J=7.2 \mathrm{~Hz}, 3 \mathrm{H}$ ); MS (ESI) $m / z$ : $444.5\left([\mathrm{M}+\mathrm{Na}]^{+}\right)$. HR-ESI-MS calcd for $\mathrm{C}_{21} \mathrm{H}_{28} \mathrm{~N}_{2} \mathrm{O}_{5} \mathrm{SNa}$ $[\mathrm{M}+\mathrm{Na}]^{+}$443.1616, found 443.1615.

\section{2 .2 化合物 6 11 的合成}

以化合物 $6 \mathbf{a}$ 和 $6 \mathbf{b}$ 的合成为例: 于 $25 \mathrm{~mL}$ 圆底烧瓶 中加入化合物 $4 \mathbf{a}(0.1 \mathrm{mmol})$, 在室温下加入 $90 \%$ 三氟醋 酸水溶液 $10 \mathrm{~mL}$, 反应 $3 \mathrm{~h}$ 后, 加碱中和反应液至中性, 二氯甲烷 $(5 \mathrm{~mL} \times 2)$ 萃取. 减压蒸除溶剂, 残留物经硅胶 (200 300 目)柱色谱分离[淋洗剂为 $V$ (二氯甲烷)： $V$ (甲 醇 $)=5: 1$ ], 得到化合物 $\mathbf{5 a}$, 收率 $92 \%$.

于 $25 \mathrm{~mL}$ 茄形烧瓶中加入化合物 $\mathbf{5 a}(40 \mathrm{mg}, 0.6$ $\mathrm{mmol}$ ), 加入 $3 \mathrm{~mL}$ 二氯甲烷(DCM)作为溶剂和干燥的三 乙胺 $\left(\mathrm{Et}_{3} \mathrm{~N}\right) 1 \mathrm{~mL}$ 、三乙烯二胺 $(\mathrm{DABCO}, 3.8 \mathrm{mg}, 0.3$ equiv.), 氮气保护, $50{ }^{\circ} \mathrm{C}$ 搅拌 $12 \mathrm{~h}, \mathrm{TLC}$ 监测反应完全. 减压蒸出 DCM, 残留物经硅胶 (200 300 目)柱色谱分 离 [淋洗剂为 $V$ (甲醇): $V$ (乙酸乙酯) $=1: 20$ ], 得到 $(13 \mathrm{a} S, 13 \mathrm{~b} R)-1,2,3,13 \mathrm{~b}$-四氢苯并 $[e]$ 吡咯并 $\left[2^{\prime}, 1^{\prime}: 3,4\right]$ 吡嗪 并 $[2,1-b][1,3]$ 噻嗪-5,8(6H,13a $H)$-二酮 (6a), 黄色固体, 三步总产率 $21 \%$. m.p. $126 \sim 127{ }^{\circ} \mathrm{C}$; $[\alpha]_{\mathrm{D}}^{25}+170.0(\mathrm{c}$ $\left.0.04, \mathrm{CH}_{3} \mathrm{OH}\right) ;{ }^{1} \mathrm{H} \mathrm{NMR}\left(600 \mathrm{MHz}, \mathrm{CDCl}_{3}\right) \delta: 8.06(\mathrm{~d}, J=$ $7.8 \mathrm{~Hz}, 1 \mathrm{H}), 7.38 \sim 7.36(\mathrm{~m}, 1 \mathrm{H}), 7.32(\mathrm{~d}, J=7.8 \mathrm{~Hz}, 1 \mathrm{H})$, $7.28(\mathrm{~d}, J=7.8 \mathrm{~Hz}, 1 \mathrm{H}), 5.51(\mathrm{~d}, J=4.2 \mathrm{~Hz}, 1 \mathrm{H}), 4.90(\mathrm{~d}$, $J=16.2 \mathrm{~Hz}, 1 \mathrm{H}), 4.30 \sim 4.27(\mathrm{~m}, 1 \mathrm{H}), 3.77(\mathrm{~d}, J=16.2 \mathrm{~Hz}$, $1 \mathrm{H}), 3.68 \sim 3.63(\mathrm{~m}, 1 \mathrm{H}), 3.60 \sim 3.56(\mathrm{~m}, 1 \mathrm{H}), 2.31 \sim 2.25$ $(\mathrm{m}, 1 \mathrm{H}), 2.24 \sim 2.18(\mathrm{~m}, 1 \mathrm{H}), 2.13 \sim 2.07(\mathrm{~m}, 1 \mathrm{H}), 2.03 \sim$ $1.97(\mathrm{~m}, 1 \mathrm{H}) ;{ }^{13} \mathrm{C}$ NMR $\left(150 \mathrm{MHz}, \mathrm{CDCl}_{3}\right) \delta: 165.9$, 164.3, 136.5, 132.2, 131.1, 129.1, 128.1, 126.5, 63.7, 56.6, 45.7, 44.7, 28.3, 24.1; HR-ESI-MS calcd for $\mathrm{C}_{14} \mathrm{H}_{14} \mathrm{~N}_{2} \mathrm{O}_{2-}$ $\mathrm{SNa}\left([\mathrm{M}+\mathrm{Na}]^{+}\right)$297.0673, found 297.0675.

按相同的方法, 以 $\mathbf{5 b}$ 为原料, 得到了相应的 $(13 \mathrm{a} R$, $13 \mathrm{~b} R)-1,2,3,13 \mathrm{~b}$ - 四氢苯并 $[e]$ 吡咯并 $\left[2^{\prime}, 1^{\prime}: 3,4\right]$ 吡嗪并 [2,1-b][1,3] 噻嗪-5,8 $(6 H, 13 \mathrm{a} H)$-二酮 $(6 \mathbf{b})$, 黄色固体, 三 步总产率 21\%. m.p. $125 \sim 127{ }^{\circ} \mathrm{C}$; $[\alpha]_{\mathrm{D}}^{25}-25.0$ (c 0.04, $\left.\mathrm{CH}_{3} \mathrm{OH}\right) ;{ }^{1} \mathrm{H}$ NMR $\left(600 \mathrm{MHz}, \mathrm{CDCl}_{3}\right) \delta: 8.12(\mathrm{~d}, J=7.8$ 
$\mathrm{Hz}, 1 \mathrm{H}), 7.41$ (t, $J=7.2 \mathrm{~Hz}, 1 \mathrm{H}), 7.29$ (t, $J=7.8 \mathrm{~Hz}, 1 \mathrm{H})$, $7.27(\mathrm{~d}, J=6.0 \mathrm{~Hz}, 1 \mathrm{H}), 4.78$ (d, $J=10.2 \mathrm{~Hz}, 1 \mathrm{H}), 4.73$ (d, $J=16.8 \mathrm{~Hz}, 1 \mathrm{H}), 4.28(\mathrm{~d}, J=17.4 \mathrm{~Hz}, 1 \mathrm{H}), 4.03 \sim 3.99(\mathrm{~m}$, $1 \mathrm{H}), 3.70 \sim 3.66(\mathrm{~m}, 1 \mathrm{H}), 3.61 \sim 3.55(\mathrm{~m}, 1 \mathrm{H}), 2.4 \sim 2.35$ (m, $1 \mathrm{H}), 2.09 \sim 2.04(\mathrm{~m}, 1 \mathrm{H}), 1.95 \sim 1.89(\mathrm{~m}, 1 \mathrm{H}), 1.76 \sim$ $1.74(\mathrm{~m}, 1 \mathrm{H}) ;{ }^{13} \mathrm{C}$ NMR $\left(150 \mathrm{MHz}, \mathrm{CD}_{3} \mathrm{OD}\right) \delta: 167.1$, 164.7, 135.5, 133.9, 131.5, 129.8, 128.5, 127.6, 62.7, 60.3, 46.9, 46.6, 31.2, 23.9; HR-ESI-MS calcd for $\mathrm{C}_{14} \mathrm{H}_{14} \mathrm{~N}_{2} \mathrm{O}_{2} \mathrm{~S}-$ $\mathrm{Na}\left([\mathrm{M}+\mathrm{Na}]^{+}\right) 297.0673$, found 297.0672.

按上述类似方法, 分别以 3-氨基丙酸甲酯盐酸盐 (2b)、 $D / L$-2-甲基甘氨酸甲酯盐酸盐( $2 \mathbf{c}$ 和 $2 \mathbf{d}$ ) 和 5 -氟颈 基水杨酸 (3b)等为原料, 得到中间体 4 后, 以其混合物 (非对映异构体)进行后续脱 Boc 和酰胺环缩合反应得到 目标产物.

$(14 \mathrm{a} S, 14 \mathrm{~b} R)-2,3,6,7$-四氢- $1 H$-苯并 $[5,6][1,3]$ 噻嗪并 [3,2-a]吡咯并 $[2,1-c][1,4]$ 二氮杂环庚熳-5,9(14a $H, 14 \mathrm{~b} H)$ 二酮 (7a): 黄色固体, 三步总产率 17\%. m.p. 133 $136{ }^{\circ} \mathrm{C} ;[\alpha]_{\mathrm{D}}^{25}+26.7$ (c $\left.0.15, \mathrm{CH}_{3} \mathrm{OH}\right) ;{ }^{1} \mathrm{H}$ NMR $(600$ $\left.\mathrm{MHz}, \mathrm{CD}_{3} \mathrm{OD}\right) \delta: 7.98(\mathrm{~d}, J=7.8 \mathrm{~Hz}, 1 \mathrm{H}), 7.45 \sim 7.42(\mathrm{~m}$, 2H), 7.33 (t, $J=7.8 \mathrm{~Hz}, 1 \mathrm{H}), 5.51$ (d, $J=1.8 \mathrm{~Hz}, 1 \mathrm{H}), 5.11$ (t, $J=7.2 \mathrm{~Hz}, 1 \mathrm{H}), 4.72 \sim 4.68(\mathrm{~m}, 1 \mathrm{H}), 3.87 \sim 3.82(\mathrm{~m}$, $1 \mathrm{H}), 3.80 \sim 3.76(\mathrm{~m}, 1 \mathrm{H}), 3.54 \sim 3.49(\mathrm{~m}, 1 \mathrm{H}), 2.83 \sim 2.75$ $(\mathrm{m}, 2 \mathrm{H}), 2.39 \sim 2.34(\mathrm{~m}, 1 \mathrm{H}), 2.27 \sim 2.22(\mathrm{~m}, 1 \mathrm{H}), 2.07 \sim$ $2.03(\mathrm{~m}, 1 \mathrm{H}), 1.93 \sim 1.88(\mathrm{~m}, 1 \mathrm{H}) ;{ }^{13} \mathrm{C}$ NMR $(150 \mathrm{MHz}$, $\left.\mathrm{CD}_{3} \mathrm{OD}\right) \delta: 171.8,166.4,137.8,133.4,131.7,130.3,129.0$, 127.6, 69.7, 56.9, 50.4, 48.9, 39.2, 37.4, 31.7, 23.5; HRESI-MS calcd for $\mathrm{C}_{15} \mathrm{H}_{16} \mathrm{~N}_{2} \mathrm{O}_{2} \mathrm{SNa}\left([\mathrm{M}+\mathrm{Na}]^{+}\right), 311.0830$, found 311.0826 .

$(14 \mathrm{a} R, 14 \mathrm{~b} R)-2,3,6,7$-四氢- $1 H$-苯并 $[5,6][1,3]$ 噻嗪并 [3,2-a]吡咯并 $[2,1-c][1,4]$ 二氮杂环庚熳-5,9(14a $H, 14 \mathrm{~b} H)$ 二酮 (7b): 黄色固体, 三步总产率 17\%. m.p. 131 $133{ }^{\circ} \mathrm{C} ;[\alpha]_{\mathrm{D}}^{25}+46.0\left(\right.$ c $\left.0.05, \mathrm{CH}_{3} \mathrm{OH}\right) ;{ }^{1} \mathrm{H}$ NMR $(600$ $\left.\mathrm{MHz}, \mathrm{CDCl}_{3}\right) \delta: 8.17(\mathrm{~d}, J=7.8 \mathrm{~Hz}, 1 \mathrm{H}), 7.41(\mathrm{t}, J=7.2$ $\mathrm{Hz}, 1 \mathrm{H}), 7.30$ (t, $J=7.2 \mathrm{~Hz}, 1 \mathrm{H}), 7.24$ (d, $J=7.8 \mathrm{~Hz}, 1 \mathrm{H})$, $4.60 \sim 4.56(\mathrm{~m}, 1 \mathrm{H}), 4.53(\mathrm{~d}, J=8.4 \mathrm{~Hz}, 1 \mathrm{H}), 4.25(\mathrm{q}, J=$ $9.0 \mathrm{~Hz}, 1 \mathrm{H}), 3.91 \sim 3.86(\mathrm{~m}, 1 \mathrm{H}), 3.47 \sim 3.45(\mathrm{~m}, 1 \mathrm{H})$, $3.41 \sim 3.36(\mathrm{~m}, 1 \mathrm{H}), 2.85 \sim 2.76(\mathrm{~m}, 2 \mathrm{H}), 2.31 \sim 2.27(\mathrm{~m}$, $1 \mathrm{H}), 1.98 \sim 1.94(\mathrm{~m}, 1 \mathrm{H}), 1.91 \sim 1.86(\mathrm{~m}, 1 \mathrm{H}), 1.67 \sim 1.62$ $(\mathrm{m}, 1 \mathrm{H}) ;{ }^{13} \mathrm{C}$ NMR $\left(150 \mathrm{MHz}, \mathrm{CDCl}_{3}\right) \delta: 171.5,162.9$, 133.0, 132.6, 130.7, 127.9, 126.9, 126.5, 66.7, 62.3, 48.8, 43.2, 38.5, 33.9, 22.4; HR-ESI-MS: calcd for $\mathrm{C}_{15} \mathrm{H}_{16} \mathrm{~N}_{2}-$ $\mathrm{O}_{2} \mathrm{SNa}\left([\mathrm{M}+\mathrm{Na}]^{+}\right), 311.0830$, found 311.0831 .

$(6 S, 13 \mathrm{a} S, 13 \mathrm{~b} R)$-6- 甲基- $1,2,3,13 \mathrm{~b}$-四氢苯并 $[e]$ 吡咯 并 $\left[2^{\prime}, 1^{\prime}: 3,4\right]$ 吡嗪并 $[2,1-b][1,3]$ 噻嗪 $-5,8(6 H, 13 \mathrm{a} H)$-二酮 (8a): 黄色固体, 三步总产率 14\%. m.p. 129 131 ${ }^{\circ} \mathrm{C}$;
$\left.[\alpha]_{\mathrm{D}}^{25}-71.0(c) 0.10, \mathrm{CH}_{3} \mathrm{OH}\right) ;{ }^{1} \mathrm{H}$ NMR $(600 \mathrm{MHz}$, $\left.\mathrm{CD}_{3} \mathrm{OD}\right) \delta: 8.05(\mathrm{~d}, J=7.8 \mathrm{~Hz}, 1 \mathrm{H}), 7.38(\mathrm{t}, J=7.5 \mathrm{~Hz}$, $1 \mathrm{H}), 7.34$ (d, $J=7.2 \mathrm{~Hz}, 1 \mathrm{H}), 7.29$ (t, $J=7.8 \mathrm{~Hz}, 1 \mathrm{H}), 5.51$ $(\mathrm{d}, J=4.2 \mathrm{~Hz}, 1 \mathrm{H}), 4.92(\mathrm{q}, J=7.2 \mathrm{~Hz}, 1 \mathrm{H}), 4.37 \sim 4.35$ (m, $1 \mathrm{H}), 3.65 \sim 3.62(\mathrm{~m}, 2 \mathrm{H}), 2.32 \sim 2.28(\mathrm{~m}, 1 \mathrm{H}), 2.76 \sim$ $2.30(\mathrm{~m}, 1 \mathrm{H}), 2.10 \sim 2.07(\mathrm{~m}, 1 \mathrm{H}), 2.02 \sim 1.97(\mathrm{~m}, 1 \mathrm{H})$, $1.58(\mathrm{~d}, J=7.2 \mathrm{~Hz}, 3 \mathrm{H}) ;{ }^{13} \mathrm{C}$ NMR $\left(150 \mathrm{MHz}, \mathrm{CDCl}_{3}\right) \delta$ : 168.2 , 164.6, 136.9, 131.9, 131.0, 129.3, 127.9, 126.4, 63.6, 55.1, 53.4, 45.0, 28.9, 23.6, 18.4; HR-ESI-MS calcd for $\mathrm{C}_{15} \mathrm{H}_{16} \mathrm{~N}_{2} \mathrm{O}_{2} \mathrm{SNa}\left([\mathrm{M}+\mathrm{Na}]^{+}\right)$311.0830, found 311.0829 .

$(6 S, 13 \mathrm{a} R, 13 \mathrm{~b} R)$-6-甲基- $1,2,3,13 \mathrm{~b}$-四氢苯并 $[e]$ 吡咯 并 $\left[2^{\prime}, 1^{\prime}: 3,4\right]$ 吡嗪并 $[2,1-b][1,3]$ 噻嗪-5,8(6H,13a $\left.H\right)$-二酮 (8b): 黄色固体，三步总产率 14\%. m.p. $129 \sim 130{ }^{\circ} \mathrm{C}$; $[\alpha]_{\mathrm{D}}^{25}+192.4\left(c \quad 0.50, \mathrm{CH}_{3} \mathrm{OH}\right) ;{ }^{1} \mathrm{H}$ NMR $(600 \mathrm{MHz}$, $\left.\mathrm{CD}_{3} \mathrm{OD}\right) \delta: 8.20(\mathrm{~d}, J=7.8 \mathrm{~Hz}, 1 \mathrm{H}), 7.42 \sim 7.38(\mathrm{~m}, 1 \mathrm{H})$, $7.31 \sim 7.26(\mathrm{~m}, 1 \mathrm{H}), 7.20(\mathrm{~d}, J=7.8 \mathrm{~Hz}, 1 \mathrm{H}), 5.60 \sim 5.50$ (m, 1H), $4.41(\mathrm{~d}, J=10.8 \mathrm{~Hz}, 1 \mathrm{H}), 3.98 \sim 3.94(\mathrm{~m}, 1 \mathrm{H})$, $3.74 \sim 3.69(\mathrm{~m}, 1 \mathrm{H}), 3.43(\mathrm{t}, J=10.8 \mathrm{~Hz}, 1 \mathrm{H}), 3.25 \sim 2.21$ (m, $1 \mathrm{H}), 2.03 \sim 1.98(\mathrm{~m}, 1 \mathrm{H}), 1.78 \sim 1.72(\mathrm{~m}, 1 \mathrm{H}), 1.62(\mathrm{~d}$, $J=7.2 \mathrm{~Hz}, 3 \mathrm{H}), 1.49 \sim 1.41(\mathrm{~m}, 1 \mathrm{H}) ;{ }^{13} \mathrm{C}$ NMR $(150 \mathrm{MHz}$, $\left.\mathrm{CD}_{3} \mathrm{OD}\right) \delta: 167.8,161.2,132.8,132.1,131.3,130.9,126.8$, 126.5, 62.6, 57.4, 53.7, 46.3, 30.8, 21.7, 16.7; HR-ESI-MS calcd for $\mathrm{C}_{15} \mathrm{H}_{16} \mathrm{~N}_{2} \mathrm{O}_{2} \mathrm{SNa}\left([\mathrm{M}+\mathrm{Na}]^{+}\right)$311.0830, found 311.0832 .

$(6 R, 13 \mathrm{a} S, 13 \mathrm{~b} R)$-6- 甲基- $1,2,3,13 \mathrm{~b}$-四氢苯并 $[e]$ 吡咯 并 $\left[2^{\prime}, 1^{\prime}: 3,4\right]$ 吡嗪并 $[2,1-b][1,3]$ 噻嗪 $-5,8(6 H, 13 \mathrm{a} H)$-二酮 (9a): 黄色固体，三步总产率 9\%. m.p. 131 133 ${ }^{\circ} \mathrm{C}$; $[\alpha]_{\mathrm{D}}^{25}-66.0\left(c \quad 0.10, \mathrm{CH}_{3} \mathrm{OH}\right) ;{ }^{1} \mathrm{H}$ NMR $(600 \mathrm{MHz}$, $\left.\mathrm{CDCl}_{3}\right) \delta: 7.97(\mathrm{~d}, J=7.8 \mathrm{~Hz}, 1 \mathrm{H}), 7.45 \sim 7.40(\mathrm{~m}, 2 \mathrm{H})$, 7.32 (t, $J=7.2 \mathrm{~Hz}, 1 \mathrm{H}), 5.65(\mathrm{~d}, J=4.2 \mathrm{~Hz}, 1 \mathrm{H}), 4.76 \sim$ $4.73(\mathrm{~m}, 1 \mathrm{H}), 4.53 \sim 4.50(\mathrm{~m}, 1 \mathrm{H}), 3.62 \sim 3.54(\mathrm{~m}, 2 \mathrm{H})$, $2.35 \sim 2.31(\mathrm{~m}, 1 \mathrm{H}), 2.23 \sim 2.17(\mathrm{~m}, 1 \mathrm{H}), 2.08 \sim 2.01(\mathrm{~m}$, $2 \mathrm{H}), 1.55(\mathrm{~d}, J=7.8 \mathrm{~Hz}, 3 \mathrm{H}) ;{ }^{13} \mathrm{C}$ NMR $(150 \mathrm{MHz}$, $\left.\mathrm{CD}_{3} \mathrm{OD}\right) \delta: 170.2,166.5,138.7,133.5,131.7,130.4,129.3$, 127.6, 64.6, 56.5, 54.6, 46.4, 29.8, 24.5, 18.2; HR-ESI-MS calcd for $\mathrm{C}_{15} \mathrm{H}_{16} \mathrm{~N}_{2} \mathrm{O}_{2} \mathrm{SNa}\left([\mathrm{M}+\mathrm{Na}]^{+}\right), 311.0830$, found 311.0833 .

$(6 R, 13 \mathrm{a} R, 13 \mathrm{~b} R)$-6- 甲基- $1,2,3,13 \mathrm{~b}$-四氢苯并 $[e]$ 吡咯 并 $\left[2^{\prime}, 1^{\prime}: 3,4\right]$ 吡嗪并 $[2,1-b][1,3]$ 噻嗪 $-5,8(6 H, 13 \mathrm{a} H)$-二酮 (9b): 黄色固体，三步总产率 9\%. m.p. $130 \sim 133{ }^{\circ} \mathrm{C}$; $[\alpha]_{\mathrm{D}}^{25}+173.0\left(c \quad 0.10, \mathrm{CH}_{3} \mathrm{OH}\right) ;{ }^{1} \mathrm{H}$ NMR $(600 \mathrm{MHz}$, $\left.\mathrm{CDCl}_{3}\right) \delta: 8.00(\mathrm{~d}, J=7.8 \mathrm{~Hz}, 1 \mathrm{H}), 7.38(\mathrm{t}, J=7.8 \mathrm{~Hz}, 1 \mathrm{H})$, $7.22 \sim 7.19(\mathrm{~m}, 2 \mathrm{H}), 5.32 \sim 5.29(\mathrm{q}, J=7.2 \mathrm{~Hz}, 1 \mathrm{H}), 4.78$ (d, $J=10.2 \mathrm{~Hz}, 1 \mathrm{H}), 3.91 \sim 3.87(\mathrm{~m}, 1 \mathrm{H}), 3.54 \sim 3.50(\mathrm{~m}$, 
$1 \mathrm{H}), 3.32(\mathrm{t}, J=10.4 \mathrm{~Hz}, 1 \mathrm{H}), 2.10 \sim 2.05(\mathrm{~m}, 1 \mathrm{H}), 1.91 \sim$ $1.87(\mathrm{~m}, 1 \mathrm{H}), 1.71 \sim 1.65(\mathrm{~m}, 1 \mathrm{H}), 1.52 \sim 1.42(\mathrm{~m}, 4 \mathrm{H})$; ${ }^{13} \mathrm{C}$ NMR (150 MHz, CD 3 OD) $\delta: 169.8,163.4,134.4$, $133.4,131.5,128.3,127.9,127.6,63.7,57.7,54.8,47.7$, 31.2, 22.6, 16.8; HR-ESI-MS calcd for $\mathrm{C}_{15} \mathrm{H}_{16} \mathrm{~N}_{2} \mathrm{O}_{2} \mathrm{SNa}$ $\left([\mathrm{M}+\mathrm{Na}]^{+}\right)$311.0830, found 311.0834 .

$(13 \mathrm{a} S, 13 \mathrm{~b} R)-11$ - 氟 $-1,2,3,13 \mathrm{~b}$ - 四氢苯并 $[e]$ 吡咯并 $\left[2^{\prime}, 1^{\prime}: 3,4\right]$ 吡嗪并 $[2,1-b][1,3]$ 噻嗪 $-5,8(6 H, 13 \mathrm{a} H)$-二 酮 (10a): 黄色固体, 三步总产率 $24 \%$. m.p. $137 \sim 138{ }^{\circ} \mathrm{C}$; $[\alpha]_{\mathrm{D}}^{25}+5.0\left(c 0.50, \mathrm{CH}_{3} \mathrm{OH}\right) ;{ }^{1} \mathrm{H}$ NMR $\left(600 \mathrm{MHz}, \mathrm{CDCl}_{3}\right)$ $\delta: 7.75(\mathrm{dd}, J=9.0,3.0 \mathrm{~Hz}, 1 \mathrm{H}), 7.30(\mathrm{dd}, J=8.4,4.8 \mathrm{~Hz}$, $1 \mathrm{H}), 7.11(\mathrm{td}, J=8.4,3.0 \mathrm{~Hz}, 1 \mathrm{H}), 5.49$ (d, $J=4.2 \mathrm{~Hz}, 1 \mathrm{H})$, $4.87(\mathrm{~d}, J=16.2 \mathrm{~Hz}, 1 \mathrm{H}), 4.30 \sim 4.27(\mathrm{~m}, 1 \mathrm{H}), 3.79$ (d, $J=$ $16.2 \mathrm{~Hz}, 1 \mathrm{H}), 3.68 \sim 3.62(\mathrm{~m}, 1 \mathrm{H}), 3.57 \sim 3.55(\mathrm{~m}, 1 \mathrm{H})$, $2.32 \sim 2.26(\mathrm{~m}, 1 \mathrm{H}), 2.22 \sim 2.17(\mathrm{~m}, 1 \mathrm{H}), 2.13 \sim 2.07(\mathrm{~m}$, 1H), $2.02 \sim 1.98(\mathrm{~m}, 1 \mathrm{H}) ;{ }^{13} \mathrm{C}$ NMR $\left(150 \mathrm{MHz}, \mathrm{CDCl}_{3}\right) \delta$ : $165.6,163.2,162.0,160.4,131.5,130.7,130.6,129.6$, 119.7, 117.6, 63.8, 56.5, 45.7, 44.7, 28.3, 24.0; HR-ESIMS calcd for $\mathrm{C}_{14} \mathrm{H}_{13} \mathrm{FN}_{2} \mathrm{O}_{2} \mathrm{SNa}\left([\mathrm{M}+\mathrm{Na}]^{+}\right)$315.0579, found 315.0576 .

$(13 \mathrm{a} R, 13 \mathrm{~b} R)-11$ - 氟 $-1,2,3,13 \mathrm{~b}$ - 四氢苯并 $[e]$ 吡咯并 $\left[2^{\prime}, 1^{\prime}: 3,4\right]$ 吡嗪并 $[2,1-b][1,3]$ 噻 嗪 $-5,8(6 H, 13 \mathrm{a} H)$-二 酮 (10b): 黄色固体, 三步总产率 24\%. m.p. 136 137 ${ }^{\circ} \mathrm{C}$; $[\alpha]_{\mathrm{D}}^{25}+27.0\left(c \quad 0.10, \mathrm{CH}_{3} \mathrm{OH}\right) ;{ }^{1} \mathrm{H} \mathrm{NMR}(600 \mathrm{MHz}$, $\left.\mathrm{CDCl}_{3}\right) \delta: 7.87(\mathrm{dd}, J=9.0,3.0 \mathrm{~Hz}, 1 \mathrm{H}), 7.26(\mathrm{dd}, J=8.4$, $3.6 \mathrm{~Hz}, 1 \mathrm{H}), 7.17$ (td, $J=8.4,3.0 \mathrm{~Hz}, 1 \mathrm{H}), 4.80$ (d, $J=10.4$ $\mathrm{Hz}, 1 \mathrm{H}), 4.77$ (d, $J=17.4 \mathrm{~Hz}, 1 \mathrm{H}), 4.25$ (d, $J=17.4 \mathrm{~Hz}$, $1 \mathrm{H}), 4.04 \sim 4.00(\mathrm{~m}, 1 \mathrm{H}), 3.73 \sim 3.70(\mathrm{~m}, 1 \mathrm{H}), 3.61 \sim 3.51$ $(\mathrm{m}, 1 \mathrm{H}), 2.41 \sim 2.37(\mathrm{~m}, 1 \mathrm{H}), 2.13 \sim 2.06(\mathrm{~m}, 1 \mathrm{H}), 2.01 \sim$ $1.91(\mathrm{~m}, 1 \mathrm{H}), 1.78 \sim 1.73(\mathrm{~m}, 1 \mathrm{H}) ;{ }^{13} \mathrm{C} \mathrm{NMR}(150 \mathrm{MHz}$, $\left.\mathrm{CDCl}_{3}\right) \delta: 164.7,162.2,161.7,160.6,130.5,128.9,128.5$, 119.9, 117.8, 62.2, 58.8, 46.3, 45.3, 30.6, 22.9; HR-ESIMS calcd for $\mathrm{C}_{14} \mathrm{H}_{13} \mathrm{FN}_{2} \mathrm{O}_{2} \mathrm{SNa}\left([\mathrm{M}+\mathrm{Na}]^{+}\right) 315.0579$, found 315.0583 .

$(14 \mathrm{a} S, 14 \mathrm{~b} R)$ - 12 -氟- $2,3,6,7$-四氢- $1 H$-苯并 $[5,6][1,3]$ 噻 嗪并 $[3,2-a]$ 吡咯并 $[2,1-c][1,4]$ 二氮杂环庚熳 5,9 $(14 \mathrm{a} H, 14 \mathrm{~b} H)$-二酮(11a): 黄色固体, 三步总产率 21\%. m.p. $145 \sim 147{ }^{\circ} \mathrm{C} ;[\alpha]_{\mathrm{D}}^{25}+44.0\left(\right.$ c $\left.0.05, \mathrm{CH}_{3} \mathrm{OH}\right) ;{ }^{1} \mathrm{H}$ NMR (600 MHz, CD $\left.{ }_{3} \mathrm{OD}\right) \delta: 7.69(\mathrm{dd}, J=9.0,3.0 \mathrm{~Hz}$, $1 \mathrm{H}), 7.48 \sim 7.45(\mathrm{~m}, 1 \mathrm{H}), 7.26(\mathrm{td}, J=8.4,3.0 \mathrm{~Hz}, 1 \mathrm{H})$, $5.52(\mathrm{~d}, J=1.8 \mathrm{~Hz}, 1 \mathrm{H}), 5.11(\mathrm{t}, J=7.8 \mathrm{~Hz}, 1 \mathrm{H}), 4.70 \sim$ $4.66(\mathrm{~m}, 1 \mathrm{H}), 3.86 \sim 3.82(\mathrm{~m}, 1 \mathrm{H}), 3.80 \sim 3.76(\mathrm{~m}, 1 \mathrm{H})$, $3.53 \sim 3.49(\mathrm{~m}, 1 \mathrm{H}), 2.86 \sim 2.74(\mathrm{~m}, 2 \mathrm{H}), 2.37 \sim 2.34(\mathrm{~m}$, $1 \mathrm{H}), 2.27 \sim 2.20(\mathrm{~m}, 1 \mathrm{H}), 2.07 \sim 2.02(\mathrm{~m}, 1 \mathrm{H}), 1.94 \sim 1.88$ $(\mathrm{m}, 1 \mathrm{H}) ;{ }^{13} \mathrm{C}$ NMR $\left(150 \mathrm{MHz}, \mathrm{CD}_{3} \mathrm{OD}\right) \delta: 171.7,165.2$,
$163.4,133.1,132.0,131.9,131.0,130.9,120.8,120.7$, $118.0,117.9,69.9,56.8,50.4,39.1,37.4,31.6,23.4$; HRESI-MS calcd for $\mathrm{C}_{15} \mathrm{H}_{15} \mathrm{FN}_{2} \mathrm{O}_{2} \mathrm{SNa}\left([\mathrm{M}+\mathrm{Na}]^{+}\right) 329.0736$, found 329.0731 .

$(14 \mathrm{a} R, 14 \mathrm{~b} R)-12$ - 氟- $2,3,6,7$ - 四氢- $1 H$ - 苯并 $[5,6][1,3]$ 噻嗪并 $[3,2-a]$ 吡咯并 $[2,1-c][1,4]$ 二氮杂环庚熳 5,9 $(14 \mathrm{a} H, 14 \mathrm{~b} H)-$ 二酮(11b): 黄色固体，三步总产率 $21 \%$. m.p. $142 \sim 145{ }^{\circ} \mathrm{C} ;[\alpha]_{\mathrm{D}}^{25}+24.0\left(c \quad 0.10, \mathrm{CH}_{3} \mathrm{OH}\right) ;{ }^{1} \mathrm{H}$ NMR $\left(600 \mathrm{MHz}, \mathrm{CDCl}_{3}\right) \delta: 7.86(\mathrm{dd}, J=9.0,2.4 \mathrm{~Hz}, 1 \mathrm{H})$, $7.23 \sim 7.21(\mathrm{~m}, 1 \mathrm{H}), 7.14(\mathrm{td}, J=8.4,3.0 \mathrm{~Hz}, 1 \mathrm{H}), 4.55$ (dd, $J=6.4,3.0 \mathrm{~Hz}, 1 \mathrm{H}), 4.53$ (d, $J=8.4 \mathrm{~Hz}, 1 \mathrm{H}), 4.24 \sim$ $4.20(\mathrm{~m}, 1 \mathrm{H}), 3.88(\mathrm{t}, J=9.6 \mathrm{~Hz}, 1 \mathrm{H}), 3.50 \sim 3.46(\mathrm{~m}, 1 \mathrm{H})$, $3.40 \sim 3.34(\mathrm{~m}, 1 \mathrm{H}), 2.83 \sim 2.75(\mathrm{~m}, 2 \mathrm{H}), 2.29 \sim 2.25(\mathrm{~m}$, $1 \mathrm{H}), 1.97 \sim 1.92(\mathrm{~m}, 1 \mathrm{H}), 1.89 \sim 1.83(\mathrm{~m}, 1 \mathrm{H}), 1.66 \sim 1.61$ $(\mathrm{m}, 1 \mathrm{H}) ;{ }^{13} \mathrm{C} \mathrm{NMR}\left(150 \mathrm{MHz}, \mathrm{CDCl}_{3}\right) \delta: 171.3,162.2$, $162.0,160.5,129.7,129.6,128.6,128.5,128.1,120.2$, 120.1, 117.4, 66.8, 62.1, 48.8, 43.2, 38.4, 33.9, 22.4; HRESI-MS calcd for $\mathrm{C}_{15} \mathrm{H}_{15} \mathrm{FN}_{2} \mathrm{O}_{2} \mathrm{SNa}\left([\mathrm{M}+\mathrm{Na}]^{+}\right)$329.0736, found 329.0732 .

\section{2 .3 抗肿瘤细胞毒性测试}

抗肿瘤细胞毒性采用改进的 Mosmann's MTT 染色 法测定 ${ }^{[22]}$. 于 96 孔板接种细胞悬液, $90 \mu \mathrm{L} /$ 孔, 细胞密 度 $2 \times 10^{4}$ 个 $/ \mathrm{mL}, 37{ }^{\circ} \mathrm{C}, 5 \% \mathrm{CO}_{2}$ 浓度的培养箱中过夜, 加 $10 \mu \mathrm{L} /$ 孔相应浓度药物 $(100 \mu \mathrm{mol} / \mathrm{L})$, 继续培 $44 \mathrm{~h}$, 加 噻唑蓝(MTT) $10 \mu \mathrm{L} /$ 孔培养 $4 \mathrm{~h}$, 从培养箱中取出, 倒 板, 加二甲基亚砜(DMSO) $100 \mu \mathrm{L} /$ 孔溶解 MTT, 测量 $570 \mathrm{~nm}$ 波长下肿瘤细胞 OD 值, 代入公式: 抑制率 $(\%)=\left(\mathrm{OD}_{\text {control }}-\mathrm{OD}_{\text {treated }}\right) / \mathrm{OD}_{\text {control }} \times 100 \%$, 计算抑制率， 顺铂为阳性对照.

辅助材料(Supporting Information) 化合物 6 11 的核 磁共振氢谱和碳谱. 这些材料可以免费从本刊网站 (http://sioc-journal.cn/)上下载.

\section{References and note}

[1] Kim, J.; Movassaghi, M. Acc. Chem. Res. 2015, 48, 1159.

[2] Amit Kumar Chattopadhyay, A. K.; Hanessian, S. Chem. Rev. 2017, $117,4104$.

[3] Chrzanowska, M.; Grajewska, A.; Rozwadowska, M. D. Chem. Rev. 2016, 116, 12369.

[4] Zi, W. W.; Zuo, Z. W.; Ma, D. W. Acc. Chem. Res. 2015, 48, 702.

[5] Tripathi, A. C.; Gupta, S. J.; Fatima, G. N.; Sonar, P. K.; Verma, A.; Saraf, S. K. Eur. J. Med. Chem. 2014, 72, 52.

[6] Zarghi, A.; Zebardast, T.; Daraie, B.; Hedayati, M. Bioorg. Med. Chem. 2009, 17, 5369.

[7] Geng, J. H.; Li, Y. X.; Zhang, W. J.; Su, X.; Guo, C. J. Shenyang Pharm. Univ. 2012, 29, 834 (in Chinese). (耿红健, 高宁, 李裕金金, 张卫军, 苏昕, 郭春, 沈阳药科大学学 报, 2012, 29, 834.)

[8] Tiwari, R.; Moraski, G. C.; Krchňók, V.; Miller, P. A.; Colon- 
Martinez, M.; Herrero, E.; Oliver, A. G.; Miller, M. J. J. Am. Chem. Soc. 2013, 135, 3539.

[9] Cao, X.; Wang, S. B.; Deng, X. Q.; Liu, D. C.; Quan, Z. S. Med. Chem. Res. 2014, 23, 1829.

[10] Feng, J. N.; Li, X. H.; Shao, J.; Zhu, M.; Li, Y.; Chen, H.; Li, X. L. Chin. J. Org. Chem. 2015, 35, 1370 (in Chinese).

(冯俊娜, 李晓慧, 郡洁, 朱墨, 李妍, 陈华, 李小六, 有机化学, 2015, 35, 1370.)

[11] Stössel, A.; Schlenk, M.; Hinz, S.; Küppers, P.; Heer, J.; Gütschow, M.; Müller, C. E. J. Med. Chem. 2013, 56, 4580.

[12] Bates, R. W. Tetrahedron Lett. 2018, 59, 559.

[13] Xiong, Y. X.; Huang, Z. S.; Tan, J. H. Eur. J. Med. Chem. 2015, 97 , 538.

[14] Manjal, S. K.; Kaur, R.; Bhatia, R.; Kumar, K.; Singh, V.; Shankar, R.; Kaur, R.; Rawal, R. K. Bioorg. Chem. 2017, 75, 406.

[15] Shao, J.; Zhu, M.; Gao, L. G.; Chen, H.; Li, X. L. Carbohydr. Res. 2018, 456,45 .
[16] Yin, Z. Q.; Zhu, M.; Wei, S. N.; Shao, J.; Hou, Y. H.; Chen, H.; Li, X. L. Bioorg. Med. Chem. Lett. 2016, 26, 1738.

[17] Chen, H.; Shao, J.; Zhu, M.; Li, X. L. Chin. J. Org. Chem. 2016 36, 527 (in Chinese). (陈华，郡洁，朱墨，李小六，有机化学，2016, 36, 527.)

[18] Jain, A. K.; Vaidya, A.; Ravichandran, V.; Kashaw, S. K.; Agrawal, R. K. Bioorg. Med. Chem. 2012, 20, 3378.

[19] Chen, H.; Yin, Q. M.; Li, C. X.; Wang, E. K.; Gao, F.; Zhang, X. B.; Yin, Z.; Wei, S. N.; Li, X. L.; Meng, M.; Zhang, P. Z.; Li, N.; Zhang, J. C. ACS Med. Chem. Lett. 2011, 2, 845.

[20] Chen, H.; Bai, J.; Jiao, L. L.; Guo, Z. H.; Yin, Q. M.; Li, X. L. Bioorg. Med. Chem. 2009, 17, 3980.

[21] CCDC-1857715 for 10a and CCDC-1857714 for 11b contain the supplementary crystallographic data for this paper. These data can be obtained free of charge from The Cambridge Crystallographic Data Centre via www.ccdc.cam.ac.uk/data_request/cif.

[22] Mosmann, T. J. Immunol. Methods 1983, 65, 55.

(Cheng, F.) 